\title{
Optimized Cutoff Value and Indication for Washout Thyroglobulin Level According to Ultrasound Findings in Patients with Well-Differentiated Thyroid Cancer
}

\author{
J.Y. Jung, J.H. Shin, B.-K. Han, and E.Y. Ko
}

\begin{abstract}
BACKGROUND AND PURPOSE: Washout thyroglobulin provides evidence for metastatic or recurrent lesions, However, the cutoff value is still controversial. We investigated an optimized cutoff value and indication for the use of washout thyroglobulin from fine-needle aspiration in neck lymph nodes from preoperative or postoperative patients with well-differentiated thyroid cancers.
\end{abstract}

MATERIALS AND METHODS: A total of 177 lymph nodes from 161 patients underwent sonographically guided fine-needle aspiration cytology and washout thyroglobulin measurement and then underwent surgery and clinical follow-up. We assessed an optimized cutoff value of washout thyroglobulin for diagnosing metastasis and compared its diagnostic performance with that of washout thyroglobulin $>10 \mathrm{ng} / \mathrm{mL}$, the currently accepted cutoff value. We also analyzed diagnostic performances of fine-needle aspiration cytology alone and of the combination of fine-needle aspiration cytology and washout thyroglobulin on the basis of the presence or absence of suspicious ultrasonographic findings.

RESULTS: Of the 177 lymph nodes, 77 were metastases and 100 were benign. An optimized cutoff value for washout thyroglobulin was 1.8 $\mathrm{ng} / \mathrm{mL}$. The sensitivity and negative predictive value in diagnosing metastasis improved significantly with a cutoff value of $1.8 \mathrm{ng} / \mathrm{mL}$ compared with a cutoff value of $10 \mathrm{ng} / \mathrm{mL}(P=.0412$ for sensitivity, $P=.0188$ for negative predictive value). In patients with suspicious ultrasonographic findings, applying washout thyroglobulin along with fine-needle aspiration cytology significantly enhanced the sensitivity and negative predictive value of fine-needle aspiration cytology performances to $100 \%$ and $100 \%$, respectively $(P=.0051$, and $P=.0088)$. There was no difference in diagnostic performance between fine-needle aspiration cytology and the combination of the 2 methods in patients without suspicious ultrasonographic findings.

CONCLUSIONS: Applying the optimized cutoff value of washout thyroglobulin of $1.8 \mathrm{ng} / \mathrm{mL}$ in patients with suspicious ultrasonographic features facilitates the diagnostic evaluation of neck lymph nodes in both preoperative and postoperative patients with well-differentiated thyroid cancer.

ABBREVIATIONS: FNA = fine-needle aspiration; FNA-Tg = washout thyroglobulin; NPV = negative predictive value; $\mathrm{PPV}=$ positive predictive value; $\mathrm{Tg}=$ thyroglobulin; US = ultrasonography

$\mathrm{H}^{\prime}$ igh thyroglobulin $(\mathrm{Tg})$ in a lymph node indicates metastatic disease in both preoperative and postoperative patients with thyroid cancer. ${ }^{1-4}$ Although this value of washout Tg (FNA-Tg) from fine-needle aspiration (FNA) serves as an important standard for suspicious recurrent or metastatic lesions, ${ }^{5}$ the cutoff value to define "high" is controversial.

In a previous report, investigators recommended that the threshold values for FNA-Tg levels be higher than $10 \mathrm{ng} / \mathrm{mL}$ if the

Received December 26, 2012; accepted after revision February 28, 2013.

From the Department of Radiology, Samsung Medical Center, Sungkyunkwan University School of Medicine, Seoul, Korea.

Please address correspondence to Jung Hee Shin, MD, Department of Radiology and Center for Imaging Science, Samsung Medical Center, Sungkyunkwan University School of Medicine, 50 Irwon-dong, Gangnam-gu, Seoul 135-710, Korea; e-mail: jhshin11@skku.edu

http://dx.doi.org/10.3174/ajnr.A3687 serum thyroglobulin level or the mean +2 SDs in node-negative patients is not available for reference. ${ }^{6}$ In real practice, some lymph nodes whose FNA-Tg values were $<10$ were tumor-involved, and the most useful cutoff value of FNA-Tg remains controversial. To increase the efficacy of the FNA-Tg measurement, one must find the standard value and the characteristics of lymph nodes for which FNA-Tg can promote more accurate results.

Generally, ultrasonography (US)-guided FNA cytology is applied to lymph nodes of patients with thyroid cancer on the basis of clinical or sonographic suspicion. ${ }^{7-9}$ Although sampling can always be done on the basis of clinical concern or an unexpected US finding, it is not cost-effective to carry out FNA-Tg on all lesions; hence, it is imperative to clarify the distinctive US characteristic that would lead to effective performance of adjunctive FNA-Tg. 
We investigated the optimal cutoff value and indication of FNA-Tg from FNA to aid the evaluation of neck lymph nodes in patients with preoperative or postoperative thyroid cancers.

\section{MATERIALS AND METHODS}

\section{Patients}

Our institutional review board approved this retrospective study and required neither patient approval nor informed consent for the review of images and medical records. All patients undergoing FNA cytology or surgery had previously received and filled out the informed consent before each procedure.

Between September 2009 and March 2010, two hundred twenty-four lymph nodes from 201 consecutive patients underwent US-guided FNA cytology and FNA-Tg from the aspiration. FNA was performed for patients with clinically suspicious nodes, dominant or enlarged nodes during routine pre-/postoperative surveillance, and/or abnormal nodes identified by another imaging technique. A total of 47 nodes were excluded due to the absence of a surgical excision or a follow-up. Thus 177 nodes from $161 \mathrm{pa}-$ tients with either a surgical excision or follow-up for at least 2 years were included in our study.

\section{Ultrasonography}

We performed ultrasonographic evaluations by using a HDI 5000 or iU22 (Philips Healthcare, Bothell, Washington) connected with a 7 - to $12-\mathrm{MHz}$ linear-array transducer. The US examinations were performed by 1 of 6 radiologists, all of whom specialized in the performance and interpretation of thyroid US for a mean of 5 years (range, 1-10). Suspicious metastatic lymph nodes on US included those that were either not oval (taller-than-wide or round) or showed microcalcifications, cystic changes, hyperechoic cortices, or irregular margins. ${ }^{10}$ We considered a US feature positive if at least 1 of these findings was shown. The size of the lymph node was measured as the largest diameter on US because the maximal axial short diameter can be subjective depending on the US operators; and describing the largest diameter of lymph nodes facilitated surgical identification of nodes and reduced confusion.

\section{Ultrasonography-Guided Fine-Needle Aspiration}

Two-milliliter syringes with 23 gauge needles attached were used for US-guided FNA cytology. The aspirates were spread on frosted-end glass slides and were immediately fixed in 95\% alcohol for both Papanicolaou and May Grunwald/Giemsa staining. In cases in which a patient had multiple lesions, we performed FNA cytology on the most suspicious or largest one. Only the most suspicious lesion from FNA-Tg measurement was chosen for evaluation. One of 7 experienced cytopathologists analyzed the slides. A typical adequate smear was one with 6 groups of $>10$ cells. Cytology for lesions was classified into 3 categories: malignant, benign, and inadequate. If the cytology showed hemosiderin-laden macrophages, we classified it as inadequate.

\section{Washout Thyroglobulin}

We used serum thyroglobulin immunoradiometric assay CT (IRMA-1; Radim, Pomezia, Italy) to measure FNA-Tg from FNA specimens. Specimens were extracted by US-guided FNA cytology
Table 1: Clinical, imaging, and cytopathologic characteristics of 177 lymph nodes

\begin{tabular}{lc}
\hline \multicolumn{1}{c}{ Characteristics } & Total $(\boldsymbol{n}=177)(\%)$ \\
\hline Age (mean) (yr) & 47 (range, 15-85) \\
Sex (female/male) & $127: 50$ \\
Mean lesion size (mean) (cm) & \\
Patient status & \\
$\quad$ Preoperative & $81(46)$ \\
$\quad$ Postoperative & $96(54)$ \\
Suspicious malignant US features & $109(62)$ \\
$\quad$ Yes & $68(38)$ \\
$\quad$ No & \\
Initial FNA & $66(37)$ \\
$\quad$ Malignant & $94(53)$ \\
$\quad$ Benign & $17(10)$ \\
Inadequate & \\
Final cytopathology & $77(44)$ \\
$\quad$ Malignant & $100(56)$ \\
Benign & \\
Reference standard & $81(46)$ \\
$\quad$ Surgical excision & $96(54)$ \\
$\quad$ Follow-up &
\end{tabular}

and smeared on the glass slides. The specimens remaining in the syringe and the needle were rinsed with $1 \mathrm{~mL}$ of normal saline and sent for the assays. The most useful cutoff value of FNA-Tg for differentiating metastasis/recurrence from benign lesions was assessed. We did not measure Tg antibodies because the clinical performance of FNA-Tg is unaffected by serum Tg antibodies. ${ }^{11}$

\section{Diagnostic Performances}

The diagnostic performances included sensitivity, specificity, positive predictive value (PPV), negative predictive value (NPV), and accuracy. We treated inadequate cytology as negative for malignancy because inadequate cytology alone does not indicate surgical excision. The diagnostic performance of the new cutoff value was compared by using FNA-Tg $>10 \mathrm{ng} / \mathrm{mL}$ as a reference standard. The performances of FNA cytology and FNA cytology with FNA-Tg at the best cutoff value were compared according to US findings. We also assessed the diagnostic performance of US findings. We investigated each cutoff value of FNA-Tg in the preoperative and postoperative state.

\section{Statistical Analyses}

The comparison of the diagnostic performances of FNA cytology and FNA cytology with FNA-Tg according with the presence or absence of suspicious US findings was analyzed by the McNemar test and Bennett method. The FNA-Tg receiver operating characteristic curve was developed by using MedCalc for Windows, Version 6.1 (MedCalc Software, Mariakerke, Belgium). The cutoff value, which maximizes the sum of the sensitivity plus specificity, was determined as the points in the upper left-hand corner. We considered a difference statistically significant with $P$ values $<.05$.

\section{RESULTS}

The clinical and cytopathologic characteristics of the 177 lymph nodes are summarized in Table 1. According to our final results, of the 177 lymph nodes, 77 (44\%) were malignant and 100 (56\%) were benign. 
The best cutoff value for FNA-Tg was $1.8 \mathrm{ng} / \mathrm{mL}$, at which the accuracy and the sum of sensitivity and specificity were highest. Sensitivity and NPV in diagnosing malignancy were significantly improved in FNA-Tg $\geq 1.8 \mathrm{ng} / \mathrm{mL}$ compared with FNA-Tg $>10$ ng/mL $(P=.0412, P=.0188$, respectively). Even though specificity and PPV were decreased by the new cutoff value, the $P$ values were not significant $(P=.4795$ and $P=.2879$, respectively) (Table 2).

A total of 109 (62\%) of 177 lymph nodes had suspicious US findings in our study. The diagnostic performance of each US finding is shown in Table 3. When at least 1 of the US findings was positive, the sensitivity, specificity, PPV, NPV, and accuracy of US findings for the diagnosis of metastasis/recurrence was $96.1 \%$, $65.0 \%, 67.9 \%, 95.6 \%$, and $78.5 \%$, respectively.

With the best cutoff value of $1.8 \mathrm{ng} / \mathrm{mL}$, the overall diagnostic performance of FNA cytology with FNA-Tg was enhanced in sensitivity and NPV while specificity and PPV decreased compared with FNA cytology alone. The statistical difference in accuracy between the combination of the 2 methods and FNA cytology alone was insignificant. Additionally, in patients with suspicious US findings, the sensitivity and NPV of FNA cytology with FNA-Tg were better than those of FNA cytology alone $(P=.0051$ and $P=.0088$ ) (Fig 1). There was no difference in diagnostic performances between FNA cytology alone and FNA cytology with FNA-Tg in patients without suspicious US findings (Fig 2

Table 2: Comparison between diagnostic performances of washout $\mathrm{Tg} \geq 1.8 \mathrm{ng} / \mathrm{mL}$ and $>10 \mathrm{ng} / \mathrm{mL}$

\begin{tabular}{lccccc}
\hline \multicolumn{1}{c}{$\%$} & Sensitivity & Specificity & PPV & NPV & Accuracy \\
\hline Washout $\mathrm{Tg}>10$ & 88.3 & 96.0 & 94.4 & 91.4 & 92.7 \\
Washout $\mathrm{Tg} \geq 1.8$ & 96.1 & 94.0 & 92.5 & 96.9 & 94.9 \\
$P$ value & .0412 & .4795 & .2879 & .0188 & .2888 \\
\hline
\end{tabular}

Table 3: Diagnostic performance of suspicious US findings for 177 lymph nodes ${ }^{a}$

\begin{tabular}{lccccc}
\hline & Sensitivity & Specificity & PPV & NPV & Accuracy \\
\hline Microcalcification & $27.3(21 / 77)$ & $95.0(95 / 100)$ & $80.8(21 / 26)$ & $62.9(95 / 151)$ & $65.5(116 / 177)$ \\
Cystic change & $28.6(22 / 77)$ & $96.0(96 / 100)$ & $84.6(22 / 26)$ & $63.6(96 / 151)$ & $66.7(118 / 177)$ \\
Hyperechoic cortex & $42.9(33 / 77)$ & $89.0(89 / 100)$ & $75.0(33 / 44)$ & $66.9(89 / 133)$ & $68.9(122 / 177)$ \\
Not oval shape & $15.6(12 / 77)$ & $86.0(86 / 100)$ & $46.1(12 / 26)$ & $57.0(86 / 151)$ & $55.3(98 / 177)$ \\
Irregular margin & $26.0(20 / 77)$ & $95.0(95 / 100)$ & $80.0(20 / 25)$ & $62.5(95 / 152)$ & $65.0(115 / 177)$ \\
At least 1 of US findings & $96.1(74 / 77)$ & $65.0(65 / 100)$ & $67.9(74 / 109)$ & $95.6(65 / 68)$ & $78.5(119 / 177)$ \\
\hline a Data are percentages. & & & & &
\end{tabular}
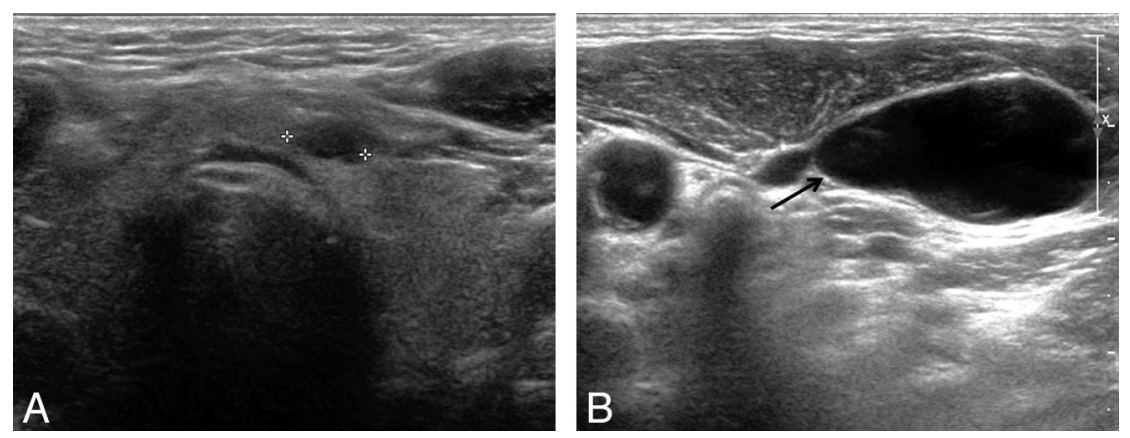

FIG 1. Ultrasonography of a 73-year-old man who had a small cystic nodule (A, cross) in the left thyroid gland and a suspicious cystic level VI lymph node ( $B$, arrow). US-guided FNA cytology was performed on the small cystic thyroid nodule and the level VI cystic node, and the results were suspicious for papillary thyroid carcinoma from a nodule and a few pigmented macrophages from a lymph node. However, metastasis was considered due to the high FNA-Tg level of $1023 \mathrm{ng} / \mathrm{mL}$. Final surgical pathology revealed a tiny papillary thyroid carcinoma of the left thyroid gland and nodal metastasis. and Table 4). Therefore, FNA-Tg was not necessary in patients with no suspicious US features. We provide a flow chart from our results (Fig 3).

According to the initial FNA cytology results of 177 lymph nodes, 66 (37\%) were malignant, 94 (53\%) benign, and 17 (10\%) inadequate.

Among the inadequate cytology cases, 8 were from too scanty cellular smear; 7, from hemosiderin-laden macrophages; 1 , from mild atypical cells; and 1, from degenerated blood. Of the 17 inadequate cytology cases, 11 were identified with surgical excision and the other 6 were confirmed as well in US follow-up of 14 months. Ten (59\%) malignant nodes were identified among 17 inadequate results. All 10 metastatic nodes had FNA-Tg levels higher than $1.8 \mathrm{ng} / \mathrm{mL}$. The remaining 7 benign nodes demontrated FNA-Tg values below $1.8 \mathrm{ng} / \mathrm{mL}$.

Of 94 initial benign cytologic results, $8(8.5 \%)$ nodes had FNA-Tg levels higher than 1.8. Two of the nodes with markedly high FNA-Tg $(>1000)$ were due to remnant thyroid tissue misinterpreted as nodes, and 2 were pathologically confirmed as metastasis. FNA-Tg of the remaining 4 cases was slightly higher than 1.8

Cytologic malignant lesions with FNA-Tg lower than 1.8 $\mathrm{ng} / \mathrm{mL}$ were found in $4(6 \%)$ of 66 cases. One of these 4 was negative for malignancy surgically and counted as false-positive FNA cytology. Subsequently, 3 of these 4 were confirmed as malignant surgically. However, these lesions showed a very low FNA-Tg $(\leq 0.6)$.

The final pathology of 161 patients was papillary thyroid carcinoma for 160 and follicular thyroid carcinoma for 1 . The best cutoff value of FNA-Tg in the preoperative and postoperative state was 1.8 and $1.9 \mathrm{ng} / \mathrm{mL}$, respectively.

\section{DISCUSSION}

Our results show that FNA-Tg can be an effective adjunct for diagnosing nodal metastasis in preoperative and postoperative patients with thyroid cancer when it is used in nodes with suspicious sonographic features. Furthermore, applying a cutoff value of $1.8 \mathrm{ng} / \mathrm{mL}$ facilitates improved sensitivity and NPV compared with the conventional standard (>10 ng/ $\mathrm{mL}$ ) when evaluating neck lymph nodes.

One prior report suggested that the most appropriate cutoff value of FNA-Tg is $1.1 \mathrm{ng} / \mathrm{mL},{ }^{12}$ and another study yielded a cutoff value of $5 \mathrm{ng} / \mathrm{mL} .{ }^{13}$ The result of our study, $1.8 \mathrm{ng} / \mathrm{mL}$, was between the 2 reports. Moreover, we demonstrate that the value of FNA-Tg was not useful in nodes without suggested suspicious US findings (Fig 3).

Given that a definitely abnormal serum $\mathrm{Tg}$ level in postoperative patients is above $2 \mathrm{ng} / \mathrm{mL},{ }^{14}$ we hypothesized that the FNA-Tg from FNA specimens would have a similar threshold value. Many radiologists tend to confuse the FNA-Tg 
cutoff, which is widely regarded as 10 , and the serum Tg level cutoff, considered as 2, leading to an increased chance of misdiagnosis. Using our cutoff value, we think that such confusion

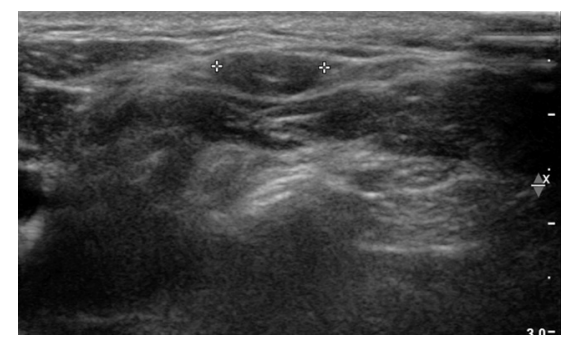

FIG 2. A 39-year-old female patient who underwent total thyroidectomy. The patient had a palpable lymph node (cross) in level $V$ and no suspicious US feature. Even though the lymph node had inadequate FNA cytology due to a few hemosiderin-laden macrophage and lymphoid cells, it was considered benign because of its low FNA-Tg level. The node showed no changes in a 2-year follow-up. According to our results, there was no significant diagnostic enhancement of FNA-Tg in patients without suspicious US findings.

Table 4: Comparison of diagnostic performances of FNA-C and FNAC with FNA-Tg according to US features using washout $\mathrm{Tg} \geq 1.8^{\mathrm{a}}$

\begin{tabular}{|c|c|c|c|c|c|}
\hline US Feature & Sensitivity & Specificity & PPV & NPV & Accuracy \\
\hline \multicolumn{6}{|l|}{ Overall } \\
\hline FNA-C & 84.4 & 99.0 & 98.5 & 89.2 & 92.1 \\
\hline $\mathrm{FNA}-\mathrm{C}+\mathrm{FNA}-\mathrm{Tg}$ & 100 & 93.0 & 91.7 & 100 & 96.1 \\
\hline$P$ value & $.0015^{\mathrm{b}}$ & $.04123^{b}$ & $.0235^{\mathrm{b}}$ & $\mathrm{b} \quad .0011^{\mathrm{b}}$ & .2386 \\
\hline \multicolumn{6}{|l|}{ Positive } \\
\hline FNA-C & 85.1 & 97.1 & 98.4 & 75.6 & 88.9 \\
\hline FNA-C+FNA-Tg & 100 & 91.4 & 96.1 & 100 & 97.3 \\
\hline$P$ value & $.0051^{\mathrm{b}}$ & .9590 & .4149 & $.0088^{\mathrm{b}}$ & .0530 \\
\hline \multicolumn{6}{|l|}{ Negative } \\
\hline FNA-C & 66.7 & 100 & 100 & 98.5 & 98.5 \\
\hline $\mathrm{FNA}-\mathrm{C}+\mathrm{FNA}-\mathrm{Tg}$ & 100 & 93.9 & 42.9 & 100 & 61.7 \\
\hline$P$ value & 1.0000 & .1336 & 02482 & .3212 & .3711 \\
\hline
\end{tabular}

Note:-FNA-C indicates FNA cytology.

a Data other than $P$ values are percentages

b Significant. between the 2 cutoffs could be reduced in a clinical setting. When we divided the patients into preoperative and postoperative states because there have been no specific mentioned reports, each cutoff value of FNA-Tg was close to 2 .

It is important that suspicious sonographic features of lymph nodes in patients with thyroid cancer be well-defined so that unnecessary FNA cytology can be avoided. Clinical suspicion such as palpable or enlarged lymph nodes during routine pre-/postoperative US surveillance leads to aspiration. However, we learned that US findings take precedence over clinical suspicion. Our results showed the high performance of our sonographic criteria for lymph node metastases in patients with thyroid cancer with a sensitivity of $96.1 \%$, specificity of 65.0 , and accuracy of $78.5 \%$. These rates were similar to the US performances of previous studies. ${ }^{15-17}$ We did not include Doppler US features because lymph nodes with abnormal vascularity frequently already have abnormal gray-scale features. As a previous report suggested, an abnormal Doppler feature alone is problematic and should serve only as a complementary finding. ${ }^{17}$

The role of FNA-Tg is very valuable when cytology is inadequate. Even though cystic change identified on US featured the highest specificity at $96.0 \%$ - but a low sensitivity of $28.6 \%$-in our results, cytology frequently showed a nondiagnostic result, such as a few hemosiderin-laden macrophages. The importance of the presence of macrophages in cystic lymph nodes has been emphasized in past research as well. ${ }^{18,19}$ In our cases, 7 of 17 inadequate results featured macrophages, and consequently all (86\%) except 1 were diagnosed as a malignancy. Therefore, FNA cytology with FNA-Tg performed on the lymph nodes that have cystic change by US findings can accurately evaluate the nodes and make up for the inadequate cytology.

Previous studies have already reported the accuracy of FNA cytology with FNA-Tg in suspicious nodal metastases, having a sensitivity and specificity ranging from $84 \%$ to $100 \%$ and $85 \%$ to $95.4 \%$, respectively. ${ }^{1,20}$ By contrast, in the overall diagnostic performance of our study with FNA-Tg $\geq 1.8$ $\mathrm{ng} / \mathrm{mL}$, consulting both FNA-Tg and FNA cytology increased the sensitivity and NPV to $100 \%$ but decreased the specificity and PPV, without any significant change in accuracy. The reason is that we considered the final result positive if the result of both or either of 2 methods was positive. Therefore, the sensitivity [truepositive/(true-positive + false-negative)] and NPV [true-negative/(true-negative + false-negative)] increased due to reduced false-negatives such as inadequate cytology, and the specificity [true-negative/ (false-positive + true-negative)] and PPV [true-positive/(true-positive + false-positive)] decreased due to increased falsepositives from the remnant thyroid tissue. The performance of FNA cytology itself was excellent in our institution (sensitiv-

FIG 3. Flow chart of recommended management of clinically suspicious lymph nodes in patients with well-differentiated thyroid carcinoma from our results. 
ity, $84.8 \%$; specificity. 99.0\%; and accuracy. 92.1\%) and thus made the value of FNA-Tg underestimated. We think that FNA cytology with FNA-Tg would be more valuable for poor performers or beginners of FNA cytology.

There are several limitations in our study. Not all of the cases were confirmed pathologically. In addition, the device for measuring FNA-Tg varies from one institution to another. Therefore, the reliability of the results may require further investigation depending on the device used. Last, interobserver variability may exist when diagnosing suspicious malignant features on the basis of US.

\section{CONCLUSIONS}

The optimal cutoff value of FNA-Tg in differentiating metastasis/ recurrence from benign lesions in patients with thyroid cancer was $1.8 \mathrm{ng} / \mathrm{mL}$. FNA cytology with FNA-Tg enhances diagnostic performance in lymph nodes with suspicious US features. Consequently, applying the optimal cutoff value and proper indication of FNA-Tg enhances diagnostic performances in the evaluation of neck lymph nodes for metastatic disease in both preoperative and postoperative patients with thyroid cancer.

\section{ACKNOWLEDGMENT}

As a researcher and the first author, J.J.Y., a 12th grade student participated in this research and demonstrated her full contribution and brilliance.

\section{REFERENCES}

1. Frasoldati A, Toschi E, Zini M, et al. Role of thyroglobulin measurement in fine-needle aspiration biopsies of cervical lymph nodes in patients with differentiated thyroid cancer. Thyroid 1999;9:105-11

2. Salmaslıoğlu A, Erbil Y, Citlak G, et al. Diagnostic value of thyroglobulin measurement in fine-needle aspiration biopsy for detecting metastatic lymph nodes in patients with papillary thyroid carcinoma. Langenbecks Arch Surg 2011;396:77-81

3. Baskin HJ. Detection of recurrent papillary thyroid carcinoma by thyroglobulin assessment in the needle washout after fine-needle aspiration of suspicious lymph nodes. Thyroid 2004;14:959-63

4. Sigstad E, Heilo A, Paus E, et al. The usefulness of detecting thyroglobulin in fine-needle aspirates from patients with neck lesions using a sensitive thyroglobulin assay. Diagn Cytopathol 2007; 35:761-67

5. Sands NB, Karls S, Rivera J, et al. Preoperative serum thyroglobulin as an adjunct to fine-needle aspiration in predicting well-differentiated thyroid cancer. J Otolaryngol Head Neck Surg 2010;39:669-73

6. Kim MJ, Kim EK, Kim BM, et al. Thyroglobulin measurement in fine-needle aspirate washouts: the criteria for neck node dissection for patients with thyroid cancer. Clin Endocrinol (Oxf) 2009; 70:145-51

7. Gharib H, Goellner JR. Fine-needle aspiration biopsy of the thyroid: an appraisal. Ann Intern Med 1993;118:282-89

8. Carmeci C, Jeffrey RB, McDougall IR, et al. Ultrasound-guided fineneedle aspiration biopsy of thyroid masses. Thyroid 1998;8:283-89

9. Danese D, Sciacchitano S, Farsetti A, et al. Diagnostic accuracy of conventional versus sonography-guided fine-needle aspiration biopsy of thyroid nodules. Thyroid 1998;8:15-21

10. Hahn SY, Shin JH, Han BK, et al. Predictive factors related to the recurrence at US-guided fine needle aspiration in postoperative patients with differentiated thyroid cancer. Clin Endocrinol (Oxf) 2011;74:270-75

11. Boi F, Baghino G, Atzeni F, et al. The diagnostic value for differentiated thyroid carcinoma metastases of thyroglobulin ( $\mathrm{Tg}$ ) measurement in washout fluid from fine-needle aspiration biopsy of neck lymph nodes is maintained in the presence of circulating anti-Tg antibodies. J Clin Endocrinol Metab 2006;91:1364-69

12. Giovanella L, Ceriani L, Suriano S. Lymph node thyroglobulin measurement in diagnosis of neck metastases of differentiated thyroid carcinoma. J Thyroid Res 2011; 2011:621839

13. Sohn YM, Kim MJ, Kim EK, et al. Diagnostic performance of thyroglobulin value in indeterminate range in fine needle aspiration washout fluid from lymph nodes of thyroid cancer. Yonsei Med J 2012;53:126-31

14. Cooper DS, Doherty GM, Haugen BR, et al. Revised American Thyroid Association management guidelines for patients with thyroid nodules and differentiated thyroid cancer. Thyroid 2009;19: $1167-214$

15. Kim E, Park JS, Son KR, et al. Preoperative diagnosis of cervical metastatic lymph nodes in papillary thyroid carcinoma: comparison of ultrasound, computed tomography, and combined ultrasound with computed tomography. Thyroid 2008;18:411-18

16. Rosário PW, de Faria S, Bicalho L, et al. Ultrasonographic differentiation between metastatic and benign lymph nodes in patients with papillary thyroid carcinoma. J Ultrasound Med 2005;24: 1385-89

17. Ahuja A, Ying M. Sonographic evaluation of cervical lymphadenopathy: is power Doppler sonography routinely indicated? $U l-$ trasound Med Biol 2003;29:353-59

18. Tseng FY, Hsiao YL, Chang TC. Cytologic features of metastatic papillary thyroid carcinoma in cervical lymph nodes. Acta Cytol 2002;46:1043-48

19. Koo JS, Kwak JY, Jung W, et al. Importance of foamy macrophages only in fine needle aspirates to cytologic diagnostic accuracy of cystic metastatic papillary thyroid carcinoma. Acta Cytol 2010;54: 249-54

20. Lee MJ, Ross DS, Mueller PR, et al. Fine-needle biopsy of cervical lymph nodes in patients with thyroid cancer: a prospective comparison of cytopathologic and tissue marker analysis. Radiology 1993; 187:851-54 